\title{
EL PATRIMONIO MARÍTIMO, LA IMPORTANCIA DE SU CONSERVACIÓN A TRAVÉS DE MUSEOS Y BUQUES
}

\author{
Ernesto Piero Bazzetti De los Santos \\ Universidad Ricardo Palma \\ pbazzetti13@hotmail.com
}

\section{RESUMEN}

Hace poco inicié un estudio para ofrecer una visión general sobre la importancia patrimonio marítimo tratando de indicar las distintas perspectivas que se tienen y los principales actores que intervienen en las mismas. Este trabajo se centrará básicamente el entender esta clasificación en el marco concreto del patrimonio, que incluirá otros elementos del patrimonio marítimo como, museos marítimos, buques museos, entre otros.

\section{PALABRAS CLAVE}

Patrimonio / Arqueología / Museo / Conservación / Turismo.

\section{ABSTRACT}

We recently lauched a study to provide an overview of the maritime heritage trying to indicate the different perspectives are taken and the main actors are involved in the sane matter. This work will be basically focus in understand this classification in the specific context of heritage, in order to kick off the sequence of items to include other elements of maritime heritage as maritime museums, museum ships, among other.

\section{KEYWORDS}

Heritage / Archeology / Community / Conservation / Tourism. 
Junto al patrimonio cultural, patrimonio artístico, patrimonio arquitectónico, etc., ha surgido en los últimos años una nueva categoría: el patrimonio marítimo. Bajo este calificativo se agrupan todos aquellos exponentes y manifestaciones de las técnicas, cultura y tradiciones marineras que la humanidad ha generado a lo largo de los siglos así como el medio natural en donde estas se desarrollan. Esto es importante, ya que la preocupación creciente por los temas medioambientales se enlaza con la consideración de lo patrimonial como un todo, natural y artificial, que debe ser preservado. La misma característica de todo aquello que tiene relación con el mar, un autentico mundo en sí mismo, ha contribuido a la creación de este concepto. Pero, para la persona ajena a las actividades del mar se trata de una cultura que puede parecer muy extraña.

Forma parte del patrimonio marítimo muchos elementos diferentes. La lista puede ser enorme, pero el denominador común es siempre el valor del objeto como testimonio de una actividad marítima. Lógicamente también hay que incluir en el patrimonio marítimo todos los vestigios de la historia marítima que se encuentran sumergidos (naufragios). Definitivamente, el patrimonio marítimo es un todo, pese a la tendencia de algunos investigadores a dividir los conocimientos. Eso es un error, ya que pocas cosas hechas por el hombre pueden ser estudiadas aisladamente, fuera de contexto.

En primer lugar, están las embarcaciones, desde el navío más grande a la más pequeña de las embarcaciones. Una nave es el reflejo de lo que una sociedad es capaz de hacer para adaptarse a su naturaleza y llevar a cabo una actividad específica. Un navío o un acorazado, pueden llegar a ser una de las máquinas más complejas que pueda construir un país, y como tal, testimonio muy rico para comprender un momento en la historia de un pueblo, por lo que no es de extrañar que se diga que "aquel país que es capaz de construir un buque de quilla a perilla sea una nación desarrollada".

Igualmente importantes, si no más, son todos los documentos referentes a la historia y a las actividades marítimas, desde un contrato de construcción naval hasta un registro de embarque que nos lleva a un cargamento concreto. Para el historiador puede ser tan útil una crónica virreinal, así como el libro de cuentas de un comerciante, de forma que no hay que valorar la importancia de un documento en función de su aparente importancia. Además, muchos documentos están hermosamente complementados con grabados que también aportan información de valor. 
También son patrimonio marítimo las estructuras arquitectónicas e industriales que tienen que ver con la navegación y las actividades marítimas, y que son muestra de la arquitectura y la ingeniería de cada nación y cada época: puertos, faros, almacenes, máquinas, etc. El desarrollo de la arqueología industrial en los últimos años ha conseguido focalizar el interés de muchos estudiosos y del gran público por este tipo de elementos. Relacionados con la técnica están también los instrumentos de navegación, las artes de pesca, las herramientas, etc. En cada uno de ellos hay a veces siglos de avances y retroceso.

Naturalmente todas las manifestaciones del arte que tienen que ver con el mar, o que encuentran en él su fuente de inspiración, pueden ser consideradas patrimonio marítimo. En pintura, incluso puede hablase de especialidades como las marinas (paisajes marítimos o costeros) o el retrato del buque en sí mismo. La escultura, la literatura, la música, las artes decorativas, la fotografía, todas tienen su lugar. También el modelismo de barcos, en todas sus formas, puede llegar a convertirse en objetos artísticos, tal como los que encontramos en los museos.

Finalmente hay algunos elementos de difícil clasificación, pero que pueden encerrar en sí mismos también la esencia de la cultura marítima: el léxico la toponimia, la gastronomía, etc. Un elemento fundamental es el patrimonio inmaterial marítimo; el conjunto de sabidurías y conocimientos acumulado durante miles de años por capitanes, marineros, carpinteros, etc. En este mismo grupo se incluyen tradiciones, supersticiones, creencias, leyendas, y cualquier otro elemento intelectual como la música, el vocabulario, las fiestas, etc. Pero por ser la gente de mar, poco dada la literatura en general, apenas han quedado testimonios escritos de muchos de estos saberes, y aunque ha habido investigadores que han recogido cuanto han podido, seguramente es mucho más lo que se ha perdido para siempre.

El patrimonio marítimo está amenazado por serios peligros en todo el mundo, pudiendo en pocos años desaparecer muchos testimonios que nos recuerdan aún miles de años de historia de la navegación. Sin embargo, en pocas décadas los cambios han sido brutales como en el siglo XX. Además de las guerras, el robo, el tráfico de arte y antigüedades y otros enemigos naturales del patrimonio cultural son peligros que inciden especialmente sobre el patrimonio marítimo. 
La desaparición de muchos oficios conlleva la pérdida de técnicas ancestrales y de un vocabulario propio. Las costas de casi todo el mundo están amenazadas por estructuras industriales o por la urbanización, con la pérdida de espacios y actividades antes dedicadas a labores marítimas. La transformación económica en el mundo de la navegación y de la pesca ha provocado la desaparición, en pocos años, de embarcaciones y artes de pesca que poco habían cambiado durante siglos. Junto a esto la revolución tecnológica ha enviado al cajón de los recuerdos infinidad de técnicas e instrumentos hasta hace poco imprescindibles. El mundo cambia a gran velocidad y todo un pasado ligado al mar puede desaparecer sin dejar rastro, empobreciendo nuestro acervo cultural y con él nuestras vidas.

Ante esto disponemos de algunas posibilidades de acción y herramientas. En primer lugar hay que señalar el importante papel de los museos marítimos como guardianes del patrimonio y de la memoria. A su lado están las instituciones y asociaciones relacionadas al mar. Se trabaja en la conservación del patrimonio y en su difusión, pero cada día más desde un punto de vista activo. Un ejemplo de dinamismo pude ser la realización de inventarios de lo que todavía existe y debe ser preservado.

El patrimonio debe ser accesible a la comunidad. Es más, ha de ser algo dinámico, vivo, que no se conserva porque sí, sino que se preserva como un elemento útil para las futuras generaciones. Afortunadamente ésta es la tendencia actual en el mundo, y la única que puede garantizar que las personas sientan interés por la cultura, la historia y la tradición. En relación con este último punto, también es importante conseguir que el patrimonio permanezca, siempre que sea posible, en su lugar original, relacionado con su medio y con la población que lo ha generado. Así resulta más sencillo darlo a conocer, sensibilizar e involucrar a la comunidad sobre la importancia de su conservación. Tenemos dos maneras de proteger el patrimonio marítimo, una es a través de los buques museo y la otra a través de los museos marítimos.

Un buque museo es aquel que ha sido conservado después de su vida activa, reacondicionado como museo es abierto al público con fines educativos, conmemorativos o turísticos. Algunas de estas naves aún pueden navegar por sus propios medios. Existen varios centenares de buques museo a nivel mundial, de diverso tipo y antigüedad, estando algunos de ellos asociados a museos navales que complementan la exposición. 
La mayoría de los buques dados de baja acaban desguazados y vendidos como chatarra; o también en el caso de las naves militares suelen ser hundidas en prácticas de tiro real. Sólo unos pocos buques acaban siendo preservados por su importancia o singularidad histórica, normalmente tras la presión de asociaciones privadas, voluntarios, entusiastas y antiguas tripulaciones. El proceso de reconversión de un buque museo suele ser largo y costoso, técnica y económicamente.

Los buques museo son herramientas para conocer la historia marítima. Estas naves son libros en los que se podría leer el pasado, aunque a veces faltasen páginas y el historiador tuviese que imaginar o reconstruir el texto perdido. El buque histórico es, ante todo, un elemento patrimonial que hay que conservar, de eso no puede caber duda en el siglo XXI. Pero tal vez valga la pena reflexionar un poco sobre el cómo y para qué.

Y es que, en lo que llamamos patrimonio marítimo, como en todos los demás campos de la cultura y sobre todo en el mundo de los museos, la conservación es básica pero no es suficiente. Los museos ya no son simples almacenes donde se guardan objetos antiguos y valiosos, sino que son lugares donde la sociedad se reencuentra con su pasado y con el producto material de su historia. Los objetos no solo se contemplan, sino que se estudian, se interpretan y se utilizan.

Cuando el objeto es un barco histórico, se plantea también la disyuntiva entre ver y utilizar, de manera que la sociedad actual no se conforma con tener un buque en un dique seco, sino que aspira a tener por ejemplo un buque del siglo XIX navegando en activo. A este deseo se unen además otros condicionantes de tipo económico, fundamentados en una explotación comercial, que permita su conservación. Es por ello que no resulta erróneo hablar de buques fijos, pasivos y activos. A veces la movilidad no es posible; imaginemos lo que supondría poner en el agua un antiguo navío y hacerlo navegar, por ello no es conveniente poner en peligro un buque de gran valor histórico, enfrentándolo a la posibilidad de perderse por un accidente en el mar.

No hay una respuesta clara sobre lo que debe ser un buque histórico, o sobre si vale la pena hacerlo navegar. Solo hay un tema de reflexión y la seguridad de que pocas cosas son más estimulantes para los amantes del mar, que un viejo barco navegando como si los años no hubieran pasado. Eso explica en gran parte el boom que se ha vivido en los últimos años, a 
escala internacional, en el campo de la recuperación de barcos antiguos y de la construcción de réplicas de embarcaciones históricas.

Hoy en día son muchas las personas e instituciones que dedican sus esfuerzos a la recuperación de buques históricos, pero resulta importante mencionar a la primera gran asociación que puso manos a la obra en este asunto y que hoy sigue siendo un punto de referencia para todos. Esta asociación es la World Ship Trust, su lema es "Nunca más" y su origen está relacionado a un hecho que hoy en día nos parece increíble e inaudito.

El 2 de diciembre de 1949, las autoridades británicas hundieron con todos los honores el HMS Implacable, un antiguo navío de línea francés llamado originalmente Duguay Trouin ${ }^{1}$. El Duguay Trouin fue un sobreviviente del combate naval de Trafalgar, en el que fue capturado y llevado al Reino Unido. Durante 144 años estuvo en servicio con la Royal Navy, pero el costo de mantenimiento era muy alto, unas 500,000 libras de la época, por lo que las autoridades decidieron su eliminación.

Aquel día muchos fueron los lamentos, y otros juraron que eso no volvería a suceder nunca más. Había que preservar los buques históricos que todavía quedaban, y había que convencer al mundo de que eran tan valiosos como muchos edificios u obras de arte.

Pronto mucha gente comprendió que valía la pena invertir en la preservación de los buques históricos, así hoy en día podemos contemplar y

1 El Duguay-Trouin, fue un navio de línea francés de la clase Téméraire, botado en 1785 en Rochefort fue incorporado en 1800. En Trafalgar estuvo al mando del capitán Claude Touffet. Capturado por los ingleses, se le dio el nuevo nombre de HMS IMPLACABLE, convirtiéndose en el primer buque en portar dicho nombre en la Royal Navy. En 1949, los británicos pretendieron devolverlo a Francia ante la imposibilidad de mantenerlo; pero el gobierno francés se negó a aceptarlo, y ante personalidades de ambas naciones fue echado a pique en el Canal de la Mancha. Desplazamiento: 1886 toneladas. Eslora: 55.20 metros. Manga: 14.90 metros. Armamento: 30 cañones de 32 libras en la cubierta baja, 30 cañones de 18 libras en la cubierta media, 12 obuses de 32 libras en la cubierta alta y 2 cañones de 12 libras en el castillo de proa. Propulsión: vela. Tripulación: 670 hombres.

Hubo un tímido intento por despertar el interés del público y evitar el sacrificio, e incluso desde la revista Illustrated London News se lanzó una campaña de concienciación, pero no tuvo éxito. Aquel ejemplar solitario, una autentica joya de la armada napoleónica, desapareció para siempre. 
admirar muchas naves en todo el mundo. El resultado de esta experiencia fue la constitución formal del World Ship Trust el 29 de diciembre de 1979, es decir 30 años después de que el IMPLACABLE fuera hundido en el Canal de la Mancha.

El registro o censo de embarcaciones es una de las herramientas qué ayudan a conocer que buques valiosos existen todavía y cuales pueden y deben ser conservados. Además del Registro que el World Ship Trust publica anualmente, debidamente actualizado, incluye los grandes buques históricos conservados en todo el mundo. Un comité técnico selecciona de entre todos los buques de interés aquellos que constituyen una muestra valiosa y significativa de la historia naval y propone cuál de ellos han de ser preservados. Naturalmente se contemplan todo tipo de buques, desde barcos de guerra hasta embarcaciones de pesca.

Otro instrumento de gran importancia es la reunión de buques históricos, afortunadamente para todos, la conservación de buques históricos y en general del patrimonio marítimo, ha pasado de ser una actividad académica para ir mucho más allá. Tal como ha pasado en otros ámbitos de la cultura, se han abierto las puertas a la participación de la gente y a un uso lúdico y alegre de la riqueza cultural de los pueblos. El resultado ha sido una nueva forma de evento nacido en países con una gran tradición marítima, que se ha ido extendiendo poco a poco por todas partes.

Por su parte los museos marítimos reúnen, conservan, estudian, interpretan y exhiben objetos y materiales relacionados con el pasado marítimo de la humanidad. Son los principales, aunque no los únicos, depositarios de una parte de la memoria colectiva de los hombres de mar de todos los tiempos y todos los rincones del planeta. Todavía hay muchos elementos patrimoniales de valor en manos de particulares, pero lo cierto es que día a día se pierden para siempre objetos o documentos irremplazables. Por fortuna siempre quedan los museos, que en la medida de sus posibilidades intentan salvar para el futuro el máximo del patrimonio.

No todos los museos marítimos del mundo son iguales y las diferencias no son solo nacionales o de tradición. Para empezar, hay museos marítimos y museos navales. Los primeros tratan la historia marítima en sus aspectos generales y son instituciones públicas o privadas, mientras que los segundos son entidades dedicadas a la guerra naval y a las armadas, es decir, de origen militar. En cada uno de los casos tanto 
el carácter como el origen de las colecciones son diferentes. Los museos navales acostumbran a nacer de colecciones reales o de fondos reunidos por algún estamento oficial (entiéndase la armada de una nación); por el contrario, lo habitual para los museos marítimos es que su nacimiento este ligado a una institución privada o en la administración civil.

También deben diferenciarse los museos generales, que explican la historia de la navegación en un lugar o en un momento, de aquellos que podríamos calificar de monográficos y que explican algún aspecto concreto como la pesca, la navegación deportiva, etc. Otra lectura la dan aquellos museos que siguen una línea argumental cronológica frente a los que se inclinan por explicar el pasado a través de grandes bloques temáticos. Finalmente también hay que hacer una distinción entre los museos que descansan su peso en la historia frente a aquellos que centran su interés en aspectos más tecnológicos y científicos.

En todos los casos resulta fundamental el valor y calidad de las colecciones, auténtica columna vertebral de un museo. Cuando un museo carece de objetos de valor o espectacularidad, debe recurrir a otro tipo de recursos (escenográficos, audiovisuales, etc.) muy útiles desde un punto de vista didáctico, pero que carecen de la magia de la pieza auténtica.

Las nuevas tendencias en cuanto a la conservación del patrimonio y a la investigación histórica precisan de nuevas formas y nuevas ideas. Es por ello que muchos museos evolucionan y buscan convertirse en algo diferente, como centros de información y documentación, servicios culturales y centros de gestión del patrimonio. Además, y no menos importante, el museo quiere convertirse en un punto de encuentro para todos aquellos que tienen interés por el patrimonio. En el caso de los museos marítimos, los amantes del mar deberían ver en el museo marítimo, un lugar donde reunirse e intercambiar experiencias, conocimientos y amistad.

Los museos marítimos del mundo, así como muchas organizaciones y personas que trabajan de forma profesional en el campo del patrimonio marítimo, están agrupados en el International Congress of Maritime Museums (ICMM), fundado en 1971 y que actúa como entidad asociada y asesora de ICOMOS. El ICMM, se reúne en un congreso cada tres años, pero entre congresos hay encuentros regionales o ciclos de conferencias.

Los museos del siglo XXI deben ser intermediarios entre los profesionales y los particulares, entre las instituciones y la sociedad. También 
deben ser el punto de encuentro entre diferentes instituciones alejadas entre sí en el espacio o a causa de su especialización; como museos, bibliotecas, parques nacionales, archivos, centros de documentación, etc. Pero no acaba aquí lo que se espera de los museos; se les pide también que presten servicios diversos, que promuevan la investigación y en definitiva, que se pongan al servicio de la sociedad.

En el Perú tenemos importantes ejemplos de preservación del patrimonio marítimo; tal vez la más vistosa sea a través de los buques museo; es así que tenemos cuatro embarcaciones convertidas en museo. En primer lugar, el submarino ABTAO en el Callao, luego tenemos a la cañonera AMÉRICA y a la motonave AYAPUA en Iquitos y finalmente al transporte YAVARÍ en Puno.

\begin{tabular}{|l|c|c|c|}
\hline \multicolumn{1}{|c|}{ NOMBRE } & TIPO & PAÍS & UBICACIÓN \\
\hline ABTAO & Submarino & Perú & Callao \\
\hline AMÉRICA & Cañonera & Perú & Iquitos \\
\hline AYAPUA & Transporte & Perú & Iquitos \\
\hline YAVARÍ & Cañonera & Perú & Puno \\
\hline
\end{tabular}

En lo referente a los museos nos encontramos con los museos administrados por la Marina de Guerra del Perú, que de manera acertada no solo se encuentran en Lima, sino en otras ciudades del Perú. Es así que tenemos el Museo Casa Grau, en Piura y Lima; los Museos Navales del Callao, Arequipa, Puno e Ilo.

\begin{tabular}{|l|l|l|c|}
\hline \multicolumn{1}{|c|}{ NOMBRE } & TIPO & PAÍS & UBICACIÓN \\
\hline Museo Casa Grau Lima & Museo & Perú & Lima \\
\hline Museo Casa Grau Piura & Museo & Perú & Piura \\
\hline Museo Naval del Callao & Museo & Perú & Callao \\
\hline Museo Naval de Arequipa & Museo & Perú & Arequipa \\
\hline Museo Naval de Ilo & Museo & Perú & Ilo \\
\hline Museo Naval de Puno & Museo & Perú & Puno \\
\hline
\end{tabular}

Adicionalmente tenemos dos museos más, poco conocidos, me refiero al Museo de la Fuerza de Aviación Naval y de la Fuerza de 
Submarinos, ambos de la Marina de Guerra del Perú, que por su alto grado de especialidad y muestra deben calificar como museos navales.

\begin{tabular}{|l|c|c|c|}
\hline \multicolumn{1}{|c|}{ NOMBRE } & TIPO & PAÍS & UBICACIÓN \\
\hline $\begin{array}{l}\text { Museo de la Fuerza de } \\
\text { Aviación Naval }\end{array}$ & Museo & Perú & Callao \\
\hline $\begin{array}{l}\text { Museo de la Fuerza de } \\
\text { Submarinos }\end{array}$ & Museo & Perú & Callao \\
\hline
\end{tabular}

Próximamente, en otras entregas, describiremos al detalle, los buques y museos que forman parte del patrimonio marítimo del Perú.

\section{REFERENCIAS BIBLIOGRÁFICAS}

BUSQUETS, Camil, Cabal, Rafael, Campanera, Albert y García, Enric. 2005 El hombre y la mar. Madrid: Aqualarga Grupo Cultural S.A.

JAFARI, Jafari.

2000 Enciclopedia del Turismo. Madrid: Editorial Síntesis S.A.

RUBIO-ARDANAZ, Juan Antonio.

2009 "El concepto de patrimonio marítimo y de cultura: gestión museística e iconos públicos en el País Vasco", Itsas Memoria. Revista de Estudios Marítimos del País Vasco, 6, Untzi Museoa-Museo Naval, Donostia-San Sebastián, pp. 57-82.

\section{Bibliografía Electrónica}

Www.patrimoniomaritimo.cl

www.untzimuseoa.eus

www.pmaritimomurcia.com

www.marina.mil.pe

www.submarinoabtao.com

www.histamar.com.ar

www.es.casamorey.com 